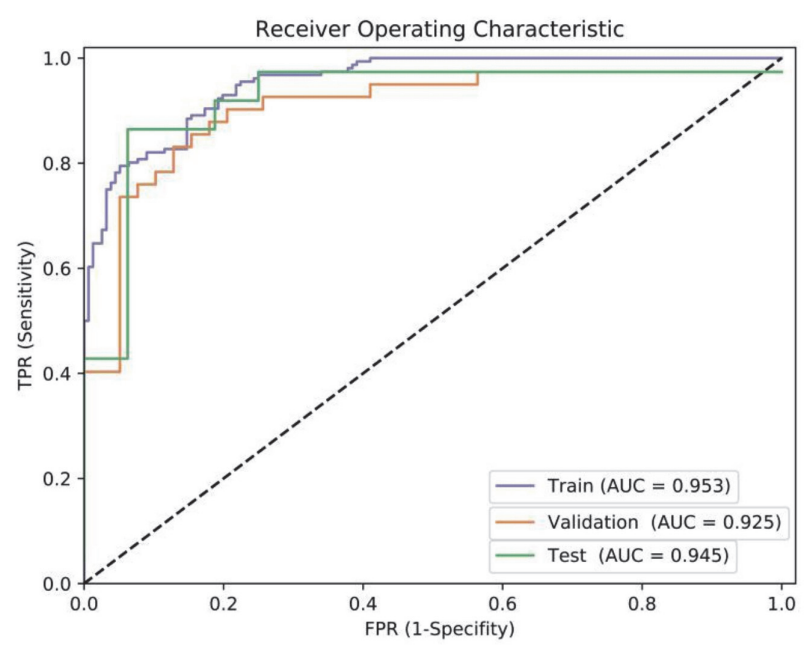

Abstract IDDF2020-ABS-0092 Figure 3 ROC curve of the clinicalradiomics model

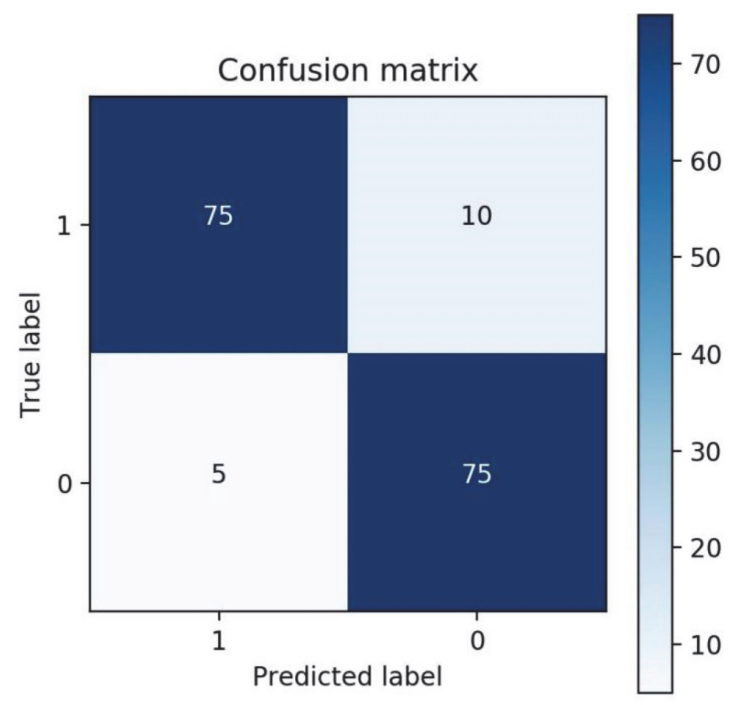

Abstract IDDF2020-ABS-0092 Figure 4 Confusion matrix of the clinical-radiomics model set and the testing set, respectively (Results are shown in figure 3 and figure 4). The model showed the maximum diameter of nodes, PLT, excision site,iMELD score and FF score were independent risk factors of PHLF in HCC patients $(\mathrm{OR}=1.258,0.986,4.670,1.237,320.382 ; \mathrm{P}<0.05$, and feature coefficients shown as figure 2). Nomogram by Logistic Regression with predictors is shown in figure 5. Moreover, the AUC of LF scoring model was significantly higher than clinical-imaging model and single radiomics model. Meanwhile, the accuracy of the model in predicting overall survival and progression-free survival of HCC patients with PHLF were 0.771 and 0.762 , respectively.

Conclusions The study indicates that LF scoring model has a good predictive value for the occurrence of PHLF in HCC patients and plays a vital role in predicting the clinical outcome of HCC patients who suffered PHLF.

\section{IDDF2020-ABS-0103 EFFICACY AND SAFETY OF PROGRAMMED CELL DEATH PROTEIN 1 INHIBITOR AND THE ASSOCIATED PROGNOSTIC FACTORS IN PATIENTS WITH HEPATITIS B VIRUS- RELATED ADVANCED HEPATOCELLULAR CARCINOMA}

Lingeng Wu*, Wei Cui, Qing Gou, Zejian Zhou. Guangong Province People's Hospital, China

\subsection{6/gutjph-2020-IDDF.153}

Background Programmed cell death protein 1 (PD1) inhibitor is safe and effective for hepatocellular carcinoma (HCC) treatment; however, the correlation between previous hepatitis $\mathrm{B}$ virus (HBV) infection and the clinical outcomes of PD1 treatment remain unclear. This study evaluated the safety and efficacy of PD-1 inhibitor treatment for HBV-related advanced HCC and determined the associated prognostic factors.

Methods Fifty HBV-infected HCC patients treated with PD-1 inhibitor in a clinical trial were retrospectively investigated. Treatment responses as per the response evaluation criteria in solid tumors 1.1 (RECIST 1.1) and immune-modified RECIST criteria (imRECIST). Overall survival (OS) and time to progression (TTP) were evaluated, and any adverse events (AEs) were recorded.

Abstract IDDF2020-ABS-0092 Figure 5 Nomogram by logistic regression with predictors 
Abstract IDDF2020-ABS-0103 Table 1 Objective responses and disease control rates between two groups per the RECIST v1.1 and imRECIST.

\begin{tabular}{|c|c|c|c|c|c|c|c|c|}
\hline & \multicolumn{4}{|c|}{ RECIST v1.1 } & \multicolumn{4}{|l|}{ imRECIST } \\
\hline & OR & NR & DC & PD & OR & NR & DC & PD \\
\hline PVTT group $(n=16)$ & & $16(100)$ & $5(31.2)$ & $11(68.8)$ & & $16(100)$ & $7(43.8)$ & $9(56.3)$ \\
\hline Non-PVTT $(n=34)$ & $4(11.8)$ & $30(88.2)$ & $18(52.9)$ & $15(44.1)$ & $5(14.7)$ & $29(85.3)$ & $19(55.9)$ & $14(41.2)$ \\
\hline$p$ value & $p=0.383$ & & $p=0.125$ & & $p=0.266$ & & $p=0.546$ & \\
\hline
\end{tabular}

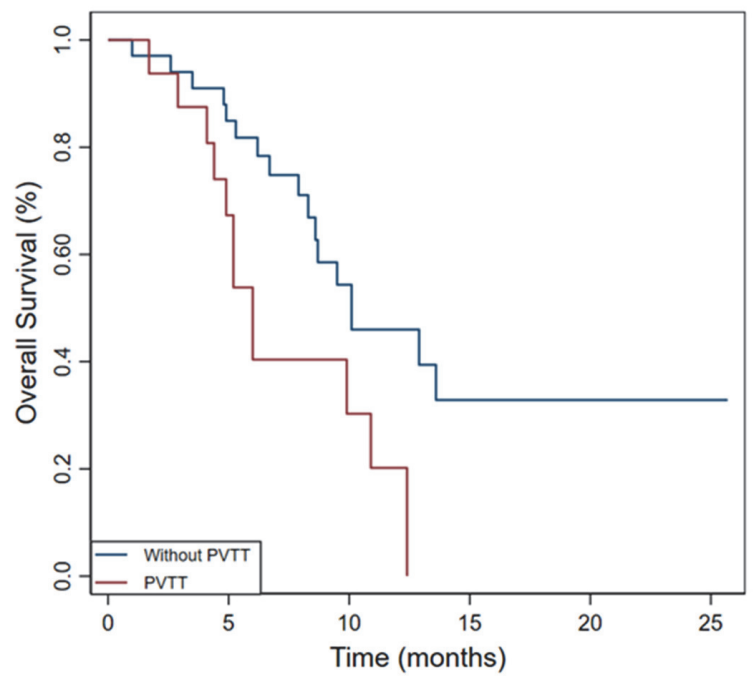

Abstract IDDF2020-ABS-0103 Figure 1 Significantly better overall survival rates were observed in the patients without portal vein tumor thrombus (PVTT) $(p=0.018)$

Results According to RECIST 1.1 criteria, no patient achieved a complete response (CR) while four (8\%) achieved partial response (PR); thus, the objective response rate (ORR) was $8 \%$. Nineteen (38\%) and $26(52 \%)$ patients exhibited stable disease (SD) and progressive disease (PD), respectively, at the first radiological assessment. The disease control rate (DCR) was $46 \%$ (table 1). The median OS was 9.5 months $(95 \%$ confidence interval [CI], 7.6-11.3), while the median TTP was 2.77 months (95\% CI, 2.1-3.5). In multivariate analysis, portal vein tumor thrombosis (PVTT) was an independent predictor of poor OS. Kaplan-Meier analysis revealed significantly shorter OS in the PVTT group than in the no PVTT group (median 6.0 vs. 10.1 months, $p=0.018$, (figure 1). HBV reactivation occurred in six patients (12\%), and the overall AEs rate was 92\%.

Conclusions PD-1 inhibitor may be safe and effective for HBV-related advanced HCC, with PVTT being a predictor of a poor prognosis.

\section{IDDF2020-ABS-0117 GUT MICROBIOTA ASSOCIATED WITH THE SENSITIVITY OF HEPATOCELLULAR CARCINOMA TO SORAFENIB}

${ }^{1}$ Weibin Lian*, ${ }^{2}$ Haiwei Li, ${ }^{3}$ Zhangran Chen, ${ }^{3}$ Yifan Lian. ${ }^{1}$ Quanzhou First Hospital Affiliated to Fujian Medical University, China; ${ }^{2}$ Liaoning Cancer Hospital and Institute, China; ${ }^{3}$ School of Medicine, Xiamen University, China

10.1136/gutjnl-2020-IDDF.154
Background Little is known about the relationship between alteration of gut microbiota and the sensitivity of hepatocellular carcinoma (HCC) to sorafenib. We performed a comparative study of gut microbiota composition between sorafenibresistant HCC patients ( $\mathrm{R}$ group, $\mathrm{n}=10$ ) and sorafenib sensitive HCC patients ( $\mathrm{S}$ group, $\mathrm{n}=10$ ).

Methods Twenty patients were classified into two groups based on the sensitivity of hepatocellular carcinoma to sorafenib within 12 months of post-sorafenib treatment. Treatment response was assessed using modified response evaluation criteria in solid tumors (mRECIST) criteria. After sorafenib treatment, the fecal samples were analyzed using 16S rRNA gene sequencing and LC-MS-based metabolomics approach.

Results Compared with the $\mathrm{R}$ group, significant gut microbiota alterations were associated with the sensitivity of HCC to sorafenib. The results showed that the $\mathrm{S}$ group had higher Faecalibacterium, Enterococcus and Veillonella abundance while the $\mathrm{R}$ group had higher levels of Lactobacillus and Prevotellaceae. Additionally, the S group had a higher bacterial network complexity compared with the $\mathrm{R}$ group. Moreover, both Salbutamol and Glyclopyramide correlated positively with Anaerostipes.

Conclusions These observations will lead to a better understanding of the relationship between alteration of gut microbiota and the sensitivity of HCC to sorafenib. Gut microbiota and microbe-associated metabolites can be used as diagnostic biomarkers in therapeutic explorations.

\section{IDDF2020-ABS-0139 GLOBAL BURDEN OF GALLBLADDER CANCER AND ITS ASSOCIATIONS WITH HDI, GDP, SMOKING, ALCOHOL DRINKING, AND OVERWEIGHT}

${ }^{1} J u n j i e$ Huang ${ }^{*},{ }^{2}$ Ping Chen, ${ }^{3}$ Shanjuan Wang, ${ }^{4}$ Lin Zhang, ${ }^{5}$ Jinqiu Yuan, ${ }^{1}$ Xiang-Qian Lao, ${ }^{1}$ Shelly LA Tse, ${ }^{6}$ Wanghong Xu, ${ }^{7}$ Zhi-Jie Zheng, ${ }^{1}$ Martin Wong. ${ }^{1} J o c k e y$ Club School of Public Health and Primary Care, Faculty of Medicine, Chinese University of Hong Kong, Hong Kong: ${ }^{2}$ Department of Gastroenterology, Ruijing Hospital North, School of Medicine, Shanghai Jiaotong University, China; ${ }^{3}$ Department of Gastroenterology, Jiading District Hospital, China; ${ }^{4}$ Melbourne School of Population and Global Health, The University of Melbourne, Australia; ${ }^{5}$ Clinical Research Centre; Scientific Research Centre, The Seventh Affiliated Hospital, Sun Yat-sen University, China; ${ }^{6}$ School of Public Health, Fudan University, China; 'Department of Global Health, School of Public Health, Peking University, China

\subsection{6/gutjpl-2020-IDDF.155}

Background This study aimed to evaluate the global incidence, mortality of gallbladder cancer, and their associations with human development index (HDI), gross domestic products (GDP), smoking, alcohol drinking, and overweight for 180 countries.

Methods The regional and national incidence and mortality figures for gallbladder cancer in 2018 were retrieved from the GLOBALCAN database. Age-standardized rates (ASRs) were evaluated by the Segi-Doll world standard population. HDI 